\title{
Frogs Jump Forward: Semantic Knowledge Influences the Perception of Element Motion in the Ternus Display
}

\author{
Patty Hsu, J. Eric T. Taylor, \& Jay Pratt \\ Version Post-print/accepted manuscript \\ Citation Hsu, P., Taylor, J.E.T., \& Pratt, J. (2015). Frogs jump forward: \\ (published version) Semantic information influences the perception of element motion in \\ the Ternus display. Perception, 44(7), 779-789. doi: \\ 10.1177/0301006615596903
Copyright/License (c) (i) $\ominus$ This work is licensed under the Creative Commons BY NC ND Attribution-NonCommercial-NoDerivatives 4.0 International License. To view a copy of this license, visit http://creativecommons.org/licenses/by-nc-nd/4.0/.

Publisher's Statement

\section{How to cite TSpace items}

Always cite the published version, so the author(s) will receive recognition through services that track citation counts, e.g. Scopus. If you need to cite the page number of the TSpace version (original manuscript or accepted manuscript) because you cannot access the published version, then cite the TSpace version in addition to the published version using the permanent URI (handle) found on the record page. 
Frogs jump forward:

Semantic knowledge influences the perception of element motion in the Ternus display

Patty Hsu, J. Eric T. Taylor, \& Jay Pratt

The University of Toronto 


\begin{abstract}
The Ternus effect is a robust illusion of motion that produces element motion at short interstimulus intervals (ISIs; $<50 \mathrm{~ms}$ ) and group motion at longer ISIs (> $50 \mathrm{~ms}$ ). Previous research has shown that the nature of the stimuli (e.g., similarity, grouping), not just ISI, can influence the likelihood of perceiving element or group motion. We examined if semantic knowledge can also influence what type of illusory motion is perceived. In Experiment 1, we used a modified Ternus display with pictures of frogs in a jump-ready pose facing forwards or backwards to the direction of illusory motion. Participants perceived more element motion with the forward facing frogs and more group motion with the backward facing frogs. Experiment 2 tested whether this effect would still occur with line drawings of frogs, or if a more life-like image was necessary. Experiment 3 tested whether this effect was due to visual asymmetries inherent in the jumping pose. Experiment 4 tested whether frogs in a "non-jumping", sedentary pose would replicate the original effect. These experiments elucidate the role of semantic knowledge in the Ternus effect. Prior knowledge of the movement of certain animate objects, in this case frogs, can also bias the perception of element or group motion.
\end{abstract}




\section{Introduction}

Our ability to perceive motion is essential for our everyday lives. From simple tasks such as pouring a cup of coffee to more complex tasks such as driving, these tasks we take for granted would not be possible without accurate motion perception. However, our visual system can also be tricked into perceiving motion when there in fact is none. Such a phenomenon occurs when we perceive words or images to be moving across billboards or other electric signs. This illusory motion is termed apparent motion, which happens when two or more static stimuli are shown in rapid succession at different locations. Rather than reporting two separate stimuli, observers perceive a single stimulus moving despite there being no actual motion (Korte, 1915; see also Ramachandran \& Anstis, 1986; Larsen, Farrell \& Bundesen, 1983). This is why we see moving text on a billboard sign as opposed to static lights flickering on and off.

Joseph Ternus, nearly nine decades ago, used this idea of apparent motion to develop the Ternus display, which produces an ambiguous illusion of motion through the use of three sequential frames (Ternus, 1926; see also Pikler, 1917). In the classic Ternus display, the first frame displays three stimuli equally spaced apart on the left side of the screen along the horizontal axis. In the second frame, no stimuli are presented, serving as the interstimulus interval (ISI). The duration of the ISI varied across the trials. On the third frame, all three stimuli are shifted to the right, such that the space occupied by the left most stimuli in the first frame is now empty. Using these three frames, subjects report seeing two different types of motion depending on the ISI. At short ISIs (e.g. < 50 ms), subjects report seeing element motion, wherein the leftmost stimulus is seen to jump to the right over the two inner stimuli, which are 
perceived as stationary. At longer ISIs (e.g. > 50 ms), subjects report seeing group motion, where all objects are seen as shifting to the right (Pantle \& Picciano, 1976). Thus, depending on the length of the ISI, participants report seeing one of two different types of motion.

While initially thought that the type of motion seen is solely dependent on ISI (Braddick \& Addlard, 1978; Braddick, 1974), several studies have shown that the nature of the stimuli is able to influence the type of motion seen. For example, Kramer and Yantis (1997) were able to increase the amount of group motion perceived by varying the degree of relatedness between their stimuli. When groups were relatively easy to form between the stimuli, subjects reported group motion at shorter ISIs relative to stimuli that were distinct to each other. A similar increase in group motion was also observed by He and Ooi (1999) as they manipulated such grouping factors as proximity and similarity. Again, when the stimuli could be easily grouped, subjects reported greater amounts of group motion across the ISIs. Other stimulus manipulations have included contrast configuration (Ma-Wyatt, Clifford, \& Wenderoth, 2005), adaptation (Petersik \& Pantle, 1979), scene-based information (using occluders to manipulate perceived size and luminance (Hein \& Moore, 2014), uniform connectedness (two elements grouped by a connecting line (Dodd, McAuley, \& Pratt, 2005), and feature information such as colours of the stimuli (Hein \& Moore, 2012). These studies have advanced the case that factors beyond ISI can influence the Ternus effect. However, the manipulations in these studies were strictly perceptual, rather than semantic. In other words, it remains unclear whether (or to what degree) semantic information can modulate the Ternus effect. 
In the current study, we wanted to determine whether higher level semantic knowledge would be able to influence the Ternus effect (i.e., can non-perceptual factors influence apparent motion in the Ternus display). Yu (2000) attempted to answer this question with two experiments where she presented participants with stimuli designed to trigger higher level semantic knowledge. In the first experiment, she presented participants with line drawings of a bus or a building. The rationale was that participants would associate more group motion with a moving bus as compared to a building. However, she did not find differences between these conditions. For her second experiment, she presented participants with stimuli depicting either a car or a person walking behind an occluder. For the car stimulus, participants could see a cab sign above the occluder, and the person's head above the occluder for the person stimulus. She found that participants reported more element motion with the person stimulus as opposed to the car stimulus, presumably because of the semantic knowledge that people can take steps whereas the wheels of cars have to stay intact and move as a group. From these experiments, she concluded that while semantic knowledge is able to influence the Ternus effect, the effect is weak and only works with certain types of semantic knowledge. However, the lack of an effect in the first experiment may be in part be due to the more abstract figurines used by Yu, an issue she herself noted. Furthermore, occlusion was used in Experiment 2 of her study, which have also been shown to influence the Ternus effect (Odic \& Pratt, 2008).

To provide a more robust test of the influence of semantic knowledge, we examined whether our knowledge of real life movements would be able to sway the type of motion we perceived with the Ternus display. Specifically, we used pictures of frogs in our experiment because of the strong association we have with frogs and "jumpiness". Anyone that has lived 
any substantial time in North America (and likely other continents as well) has seen frogs jumping, either in real life or through various depictions, and our association of them jumping has even led to the creation of the game "leap frogs". Importantly for our study, the morphology of frogs is such that they can only jump in a forward direction, and we have no association with frogs leaping backwards. Taking advantage of this morphological asymmetry, we hypothesize that due to our prior knowledge that frogs only jump forwards, more element motion would be seen using pictures of frogs that faced in the direction of the illusory motion as compared to pictures of frogs that faced backwards to the illusory motion.

\section{Experiment 1}

We used a classic three frame Ternus display with pictures of frogs instead of the traditional geometric stimuli. One condition (forward facing) had both frogs facing in the same direction as the apparent motion. In the other condition (backward facing), both frogs were shown to be facing opposite to the direction of the apparent motion. For rightward element motion to occur in this condition, the left-most frog would have to jump a considerable distance backwards over the center frog. If element motion is modulated by semantic knowledge of the frogs jumping abilities, the backward facing frogs should elicit less element motion and more group motion compared to the forward facing frogs. 


\section{Methods}

\section{Subjects}

Nine undergraduate students enrolled in PSY100 at the University of Toronto volunteered to participate in the one hour experiment and received course credit for their participation. All subjects had normal or corrected-to-normal vision and were naïve about the purpose of the experiment.

\section{Procedure}

Images of two frogs (one at fixation, the other $6.2^{\circ} \mathrm{left}$ ) were presented for $500 \mathrm{~ms}$ (see Figure 1) on a white background (RGB: $255,255,255)$. The two frogs were either both facing to the right (forward facing) or both facing to the left (backwards facing). Next, a blank screen appeared for one of ten ISIs $(0,12,24,36,48,60,72,84,96$, or $108 \mathrm{~ms})$. Following the blank ISI the same two stimuli reappeared at different locations, with one at fixation and the other to the right $6.2^{\circ}$. After the two stimuli reappeared, subjects indicated whether they perceived element motion ("z" key) or group motion ("/" key) on the keyboard. Prior to the experimental trials, subjects performed 20 practice trials with pairs of grey squares as stimuli (1 pixel border, RBG of 128, 128, 128; interior RBG of 192, 192, 192). Practice trials displayed ten trials at a 12 ms ISI for an example of element motion and another ten trials at a $108 \mathrm{~ms}$ ISI as an example of group motion. The subject's heads were held stable by a chin and head rest placed $44 \mathrm{~cm}$ from the front of the computer monitor. 


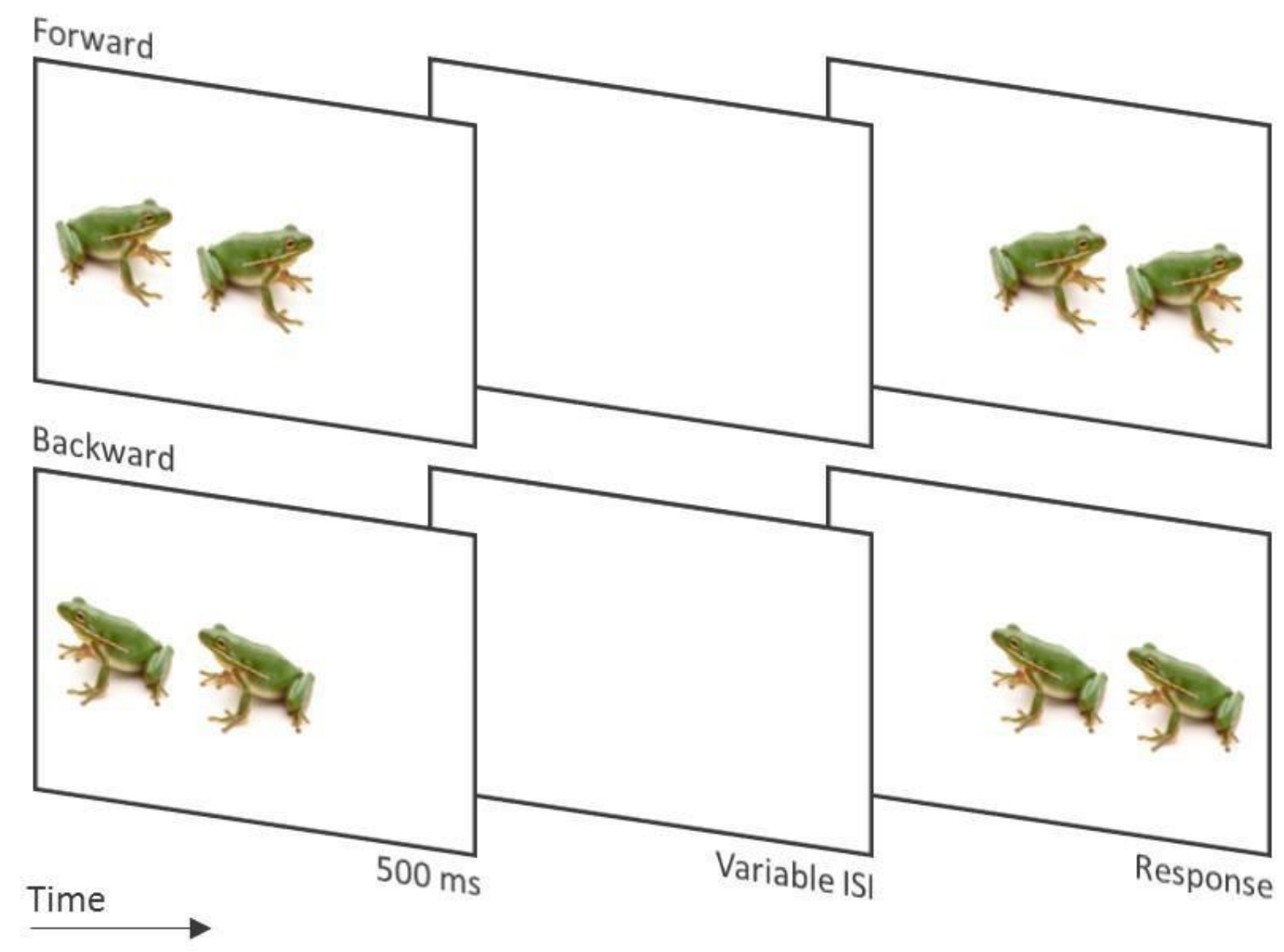

Figure 1. Time course for a given trial. Both direction conditions are shown. The time course was the same for all experiments; only the stimuli changed. The ISI ranged from $0 \mathrm{~ms}$ to $108 \mathrm{~ms}$.

Design

Each subject completed 480 trials, with short breaks every 80 trials. The directionality of the stimuli and the 10 ISIs were fully randomized throughout the experiment.

\section{Results and Discussion}

The percentage of element motion reported by subjects was plotted against ISI and is presented in Figure. 2. The data were analyzed using a 2 (direction of frogs: forward or 
backwards) $\times 10$ (ISI) analysis of variance (ANOVA). As expected, there was a significant main effect of ISI, $F(9,72)=2.957, p<.01, \eta_{p}^{2}=.270$, with a greater proportion of element motion seen at shorter ISIs compared to longer ISIs. This is the classic ambiguous apparent motion of the Ternus display. Importantly, there was also a significant main effect of direction, $F(1,8)=$ $7.204, p<.028, \eta_{p}^{2}=.474$, with the perception of more element motion with the forward facing frogs relative to the backward facing frog. There was no interaction between ISI and direction of stimuli, $F(9,72)<1$.

The main effect of stimulus direction on the likelihood of perceived element motion is consistent with the way frogs jump. When the frogs were oriented in a direction compatible with frog jumping (forward), the likelihood of perceived element motion increased compared to when the frogs were oriented in a direction that would make jumping impossible. This is not the first demonstration of semantic knowledge influencing the Ternus effect. However, Yu's (2000) earlier experiments produced small effects of semantic knowledge on apparent motion in the Ternus display. It is possible that our results exaggerated the effect of semantic knowledge on the Ternus effect because the stimuli were realistic photographs of frog stimuli, as opposed to Yu's line drawings. To test whether the effect of semantic information on the Ternus effect is modified by image quality, we conducted a second experiment replicating Experiment 1 using line drawings of the same frogs. 

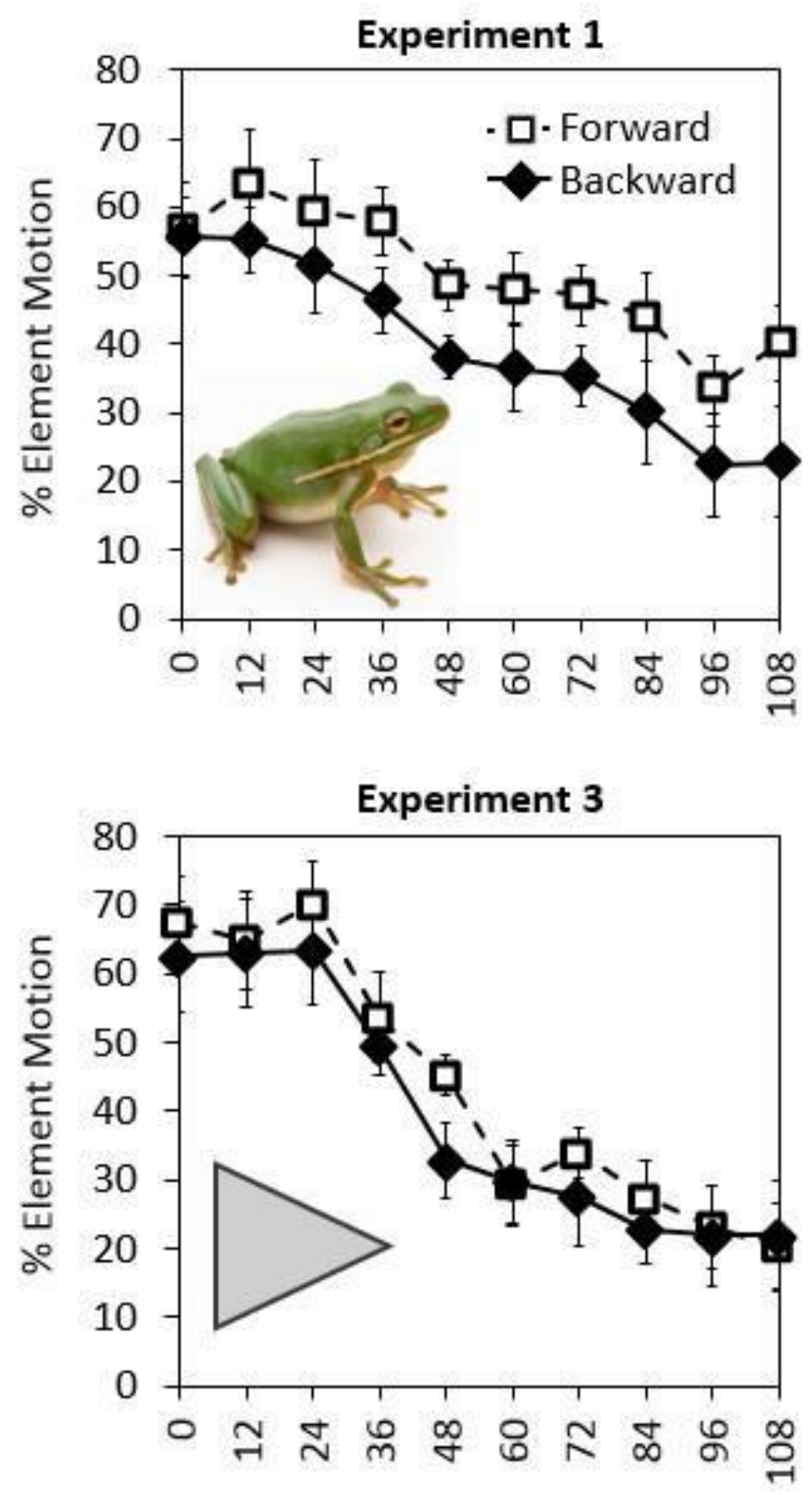

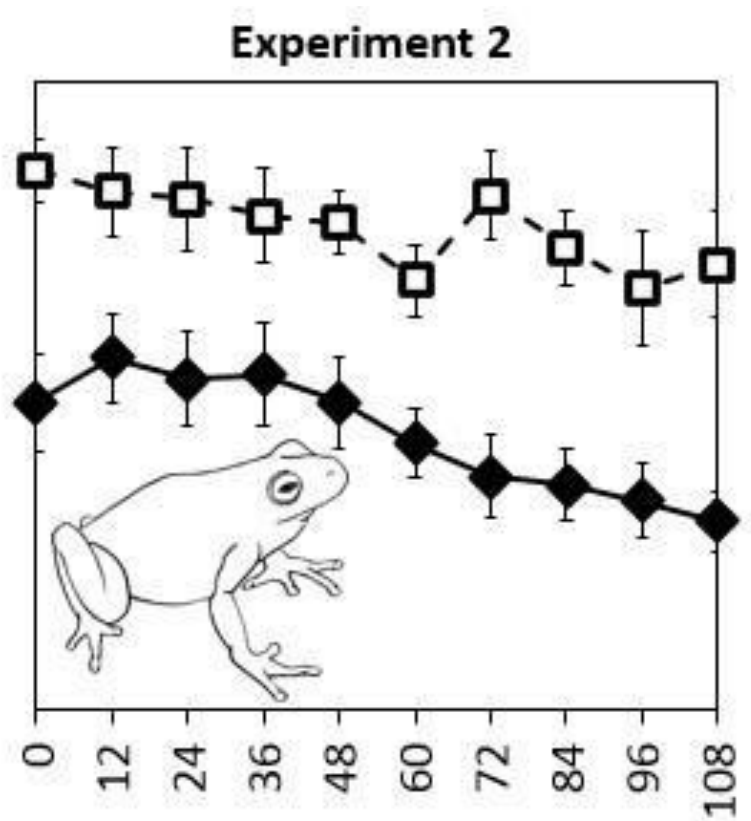

Experiment 4

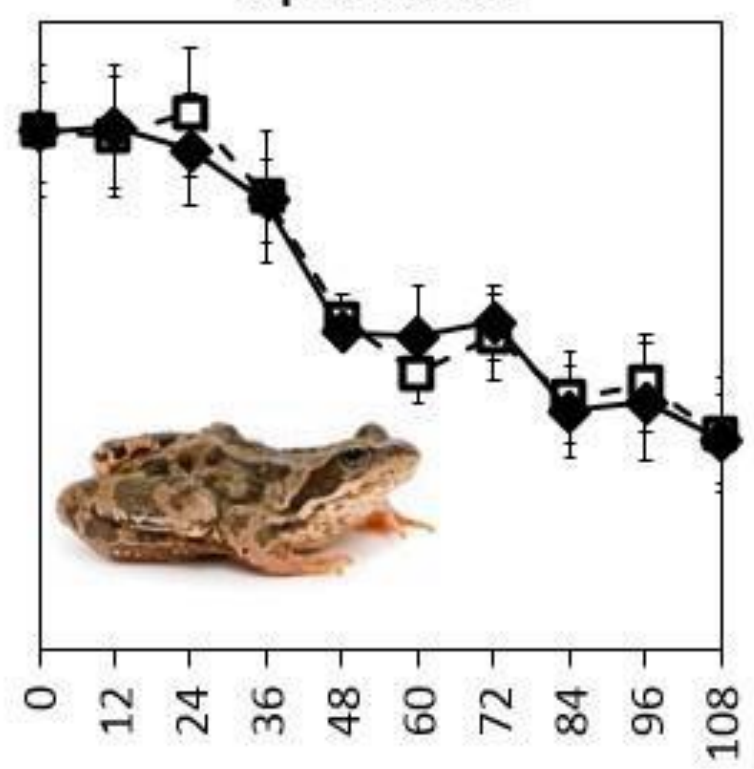

ISI (ms)

Figure 2. Perceived element motion plotted against ISI for all four experiments. Stimuli are displayed within each graph. Error bars represent within-subject standard error of the mean. 


\section{Experiment 2}

We attributed the effect of frog direction on perceived element motion in the first experiment to the semantic knowledge that frogs only jump forward. To further test this idea, we used line drawings of the same frogs in Experiment 2. If semantic knowledge about frogs' actions truly drove the effect observed in Experiment 1, then the image quality shouldn't matter; any schematic images of frogs ready to jump should elicit the same effect on perceived element motion (i.e., more group motion with forward facing frogs than backward facing frogs).

\section{Methods}

Subjects

Eight students from the University of Toronto volunteered or were compensated $\$ 10$ to participate. All subjects had normal or corrected-to-normal vision and were naïve about the purpose of the experiment.

Procedure and Design

The procedure and design of the second experiment was identical to the first experiment except for the stimuli presented. Outlines of the frog images from Experiment 1 were traced and scanned. Using GIMP image manipulation software, we removed all information except for the traced lines, leaving us with line drawings of the same frogs from Experiment 1 (see Figure 2). 


\section{Results and Discussion}

We repeated the analysis used in Experiment 1. Once again, we observed a Ternus effect, as there was a significant main effect of ISI, $F(9,63)=2.59, p=.013, \eta^{2}{ }_{p}=.270$, with a greater proportion of element motion seen at shorter ISIs compared to longer ISIs. We also replicated the main effect of direction, $F(1,7)=13.47, p=.008, \eta_{p}^{2}=.658$, as forward facing frogs were perceived with more element motion than backwards frogs. There was no interaction between the factors, $F(9,63)=1.09$. Because we replicated Experiment 1 with visually impoverished images of the same frogs, we are confident that the effect is driven by non-visual factors; specifically, the knowledge that frogs jump forward affected the likelihood of perceiving element motion. However, semantic knowledge of how frogs jump is confounded with the direction they face (i.e., frogs have a clearly defined front and back). It may be possible that the visual asymmetries of the frog stimuli biased perception in some manner as to influence group motion. Therefore, we conducted a third experiment with stimuli that were similarly asymmetric, but totally inanimate.

\section{Experiment 3}

To determine whether it was simply the directionality of the frog or the higher level semantic knowledge of the frog itself creating the effect in the first two experiments, we used inanimate triangles facing forwards or backwards in the third experiment. The triangles, similar to the frogs, had distinct visual asymmetries and thus could produce the same effect as the frog stimuli if the effect is simply caused by directionality. Moreover, they could not possibly be mistaken for frogs. This control experiment is important because simple static image elements, 
such as motion lines (lines trailing an object, indicating movement in the opposite direction) can influence motion perception of static images (Kawabe \& Miura, 2008). Moreover, object asymmetries can affect ambiguous motion illusions (McBeath, Morikawa, \& Kaiser, 1992; Proffitt, Gilden, Kaiser, \& Whelan, 1988). Consequently, we wanted to test whether simple visual asymmetries could account for the effect described in Experiments 1 and 2.

\section{Methods}

Subjects

Eight undergraduate students enrolled in PSY100 at the University of Toronto volunteered to participate in a one hour experiment and received course credit for their participation. All subjects had normal or corrected-to-normal vision and were naïve about the purpose of the experiment.

Procedure and Design

The procedure and design of the second experiment was identical to the first experiment except for the stimuli presented. In this experiment two triangles were presented either facing forwards or backwards (see Figure 2).

\section{Results and Discussion}

We repeated the analysis used in Experiment 1. Once again, we observed a Ternus effect, as there was a significant main effect of ISI, $F(9,63)=8.188, p<.001, \eta^{2}{ }_{p}=.511$, with a greater proportion of element motion seen at shorter ISIs compared to longer ISIs.

Importantly, in contrast with the previous experiment, there was not a significant main effect of 
direction, $F(1,7)=1.322, p<0.288$, with equal amounts of element motion perceived with the forward facing stimuli relative to the backward facing stimuli (see Figure 1). There was no significant interaction between ISI and size of stimuli, $F(9,63)<1$. Unlike Experiments 1 and 2, the directionality of the triangles themselves did not produce more element motion when facing forwards as compared to facing backwards, indicating that visual asymmetries cannot account for the strong group motion perceived with frogs.

We chose isosceles triangles as our control objects because they are maximally directional, clearly defining a front and back side. However, there are elements other than directionality which might conceivably affect apparent motion. For example, the jump-ready posture of the frogs in Experiment 1 might be best defined by the angle of the frog's body to ground, rather than directionality, and our vertically symmetrical triangles may not have captured this imbalance ${ }^{1}$. Simple image elements can, after all, influence the perception of apparent motion (e.g. Kawabe \& Miura, 2008; Proffitt et al., 1988). However, McBeath et al. (1992) showed that horizontally-oriented isosceles triangles (exactly like our triangles) were optimally configured to affect apparent motion as compared to several other shapes, several of which possessed the vertical asymmetries not unlike our frogs. Most importantly for our purposes, McBeath et al. (1992) demonstrated that their triangles had a stronger influence on apparent motion than drawings of biological figures that possessed a clear angle between body and ground (specifically the running "stickman" stimulus from their second experiment). Thus, if the slant of the frogs' posture caused the observed differences in group versus element motion, we can

\footnotetext{
${ }^{1}$ We thank an anonymous reviewer for raising this issue.
} 
reasonably expect that the horizontally-oriented isosceles triangles should have had a greater

effect. It would be ideal to control for the influence of basic image statistics on apparent motion by presenting a control image with the same image statistics, but that is not interpreted as a frog. However, we contend that to do so is practically impossible, as the image statistics of a frog are perfectly confounded with it being an image of a frog.Experiment 4

In the first two experiments we found that forward facing frogs produced more element motion as compared to backward facing frogs. The frogs selected in the first two experiments appeared in a ready-to-jump pose which was deliberately chosen to facilitate the ease of jumping association. In this fourth experiment, we selected frogs who appeared were resting and lying down while still having a distinct front end and backend. In other words, although these stimuli represented animate objects with fronts and backs, these animals were not prepared to jump. This experiment will determine if asymmetrical animate images are sufficient to alter the Ternus effect.

\section{Methods}

\section{Subjects}

Seven undergraduate students enrolled in PSY100 at the University of Toronto volunteered to participate in the one hour experiment and received course credit for their participation. All subjects had normal or corrected-to-normal vision and were naïve about the purpose of the experiment.

\section{Procedure and Design}


The procedure and design were very similar to the previous experiments except for the stimuli used (see Figure 2). Rather than using ready to jump frogs or triangles, frogs who appeared to be lying down and resting were presented either in a forward facing direction or a backwards facing direction.

\section{Results and Discussion}

We repeated the analysis used in Experiment 1. As expected, there was a significant main effect of ISI, $F(9,54)=6.278, p<.001, \eta_{p}^{2}=.539$, with a greater proportion of element motion seen at shorter ISIs compared to longer ISIs. However, there was no significant main effect of directionality, $F(1,6)<1$, nor any significant interaction between ISI and frame $F(9,54)$ $<1$ (see Figure 1). Thus, resting state frogs did not produce more element motion when facing forwards as compared to ready-to-jump frogs in Experiment 1 and 2.

\section{General Discussion}

The present experiments demonstrates the effect of higher level semantic knowledge on the Ternus effect; in this particular case, our knowledge of frogs and their bias to jump forwards and not backwards. In Experiment 1, we manipulated the directionality of the frogs and found that frogs that were facing forwards resulted in greater element motion as compared to frogs that were facing backwards. In Experiment 2, we used line drawings of the same frog used in Experiment 1 and found the same effect as in Experiment 1. This suggests that semantic knowledge of the frogs, regardless of image quality of the stimuli, produces the effect. In Experiment 3, we wanted to determine whether directionality itself, without the influence of our semantic knowledge of animacy, would be sufficient to produce more element 
motion. However, we found that using inanimate triangles that had distinct front and back ends rather than pictures of frogs was not enough to produce the effect. In Experiment 4, we used pictures of frogs that appeared to be in a resting state, and found that directionality and animacy are not sufficient, in and of themselves, to produce an effect of greater element motion.

The main finding of the current study is that object-specific knowledge influences the type of motion participants perceived with the Ternus paradigm. Specifically, the knowledge that frogs jump forward affected the type of apparent motion. This finding coincides with a previous study done by Shiffrar and Freyd (1990) who showed that knowledge of human motion influenced the perception of apparent motion paths. They showed participants two still frames of bodies in different positions and varied the ISI. Critically, the two frames depicted bodies in positions where the shortest path of motion between positions would be impossible. They found that at short ISIs, the impossible motion was perceived, whereas at longer ISIs, participants perceived the biologically plausible apparent motion. They concluded that when given a sufficient amount of time and appropriate stimuli, participants tend to perceive objectappropriate apparent motions.

Our first two experiments used frog stimuli, capable of triggering frog-specific knowledge and thus led to the effect seen. However, the images of simple asymmetric triangles in our third experiment were not enough to produce an effect, despite having a distinct directionality. Geometric triangles are not associated with any object-specific knowledge as they are so common and widely seen in everyday life. Therefore, we concluded that rather than 
directionality itself, it was the object-specific knowledge of the frogs' jumping motions which influenced the type of motion perceived. In addition, our object-specific knowledge about resting frogs also inform us that resting frogs do not afford jumping as easily as those that are in a ready-to-jump state, which resulted in no difference between frogs that were facing forwards or facing backwards in a resting state. Therefore, our previous semantic knowledge regarding frogs and lack of semantic knowledge regarding triangle-specific motion resulted in the effects or null-effects seen. However, the influence of semantic knowledge on the Ternus display is rather surprising, as it is essentially influencing our perception of motion when there is no actual motion to be perceived. This highlights how strong our top-down visual system is and how persistent its influences are.

It is important to note that the present study obtained much larger effects than that of Yu (2000). While it may be argued that the more life-like frog photos chosen in the experiments were more life-like than the abstract line drawings chosen by Yu, Experiment 2 similarly used line drawings of frogs and still produced the same result. Though the line drawings chosen by Yu may have still been more abstract than the stimuli used in Experiment 2 (e.g. cartoon line drawings versus life-like line drawings), it suggests that image quality was not a factor. Rather, the semantic information elicited by the frogs was sufficient to produce the effect. This may be due to the fact that frog "jumpiness" is so engrained and automatically associated, that a clear image was not necessary. This may explain why Yu (2000) found smaller effects, as motion is not typically the first thing we would associate with buildings or taxis (stimuli used in the experiments). Because of the weaker associations, more life-like images may have been needed to produce significant effects. 
One outstanding issue is whether the effect of frog orientation on perceived element motion represents an enhancement or a decrement. Specifically, it is unclear whether forwardfacing frogs are perceived with more element motion relative to a baseline or whether backward-facing frogs are perceived with less. It is unclear how to select an appropriate baseline against which the frog motion can be judged. Consequently, we remain agnostic on this issue, although the conclusion is fundamentally the same either way: Semantic knowledge about the way things move affects the Ternus illusion.

The results of the present experiments agree with other findings showing that the Ternus effect does not depend solely on the ISI, but also on the nature of the stimuli. The fact that many experiments have manipulated the nature of the stimuli in different ways - with perceptual grouping (He \& Ooi, 1999; Kramer \& Yantis, 1997), uniform connectedness (Dodd et al., 2005), feature information (Hein \& Moore, 2012), scene-based information (Hein \& Moore, 2014) - and have all successfully disrupted the normal Ternus effect lends support to the idea that the nature of the stimuli is a key component in the Ternus displays. These experiments, along with the current study, provide evidence that the effect is not simply due to the time between the object displays, but instead various perceptual and higher level cognitive information can affect what illusory motion is perceived. 


\section{References}

Braddick, O. (1974). A short-range process in apparent motion. Vision Research, 14(7), 519-527.

Dodd, M. D., McAuley, T., \& Pratt, J. (2005). An illusion of 3-D motion with the Ternus display. Vision Research, 45(8), 969-973.

He, Z. J., \& Ooi, T. L. (1999). Perceptual organization of apparent motion in the Ternus display. Perception, 28(7), 877-892.

Hein, E., \& Moore, C. M. (2012). Spatio-temporal priority revisited: The role of feature identity and similarity for object correspondence in apparent motion. Journal of Experimental Psychology, 38(4), 975-988.

Hein, E., \& Moore, C. M. (2014). Evidence for scene-based motion correspondence. Attention, Perception, \& Psychophysics, 76, 793-804.

Kawabe, T., \& Miura, K. (2008). New motion illusion caused by pictorial motion lines. Experimental psychology, 55(4), 228.

Korte, A. Kinematoskopische untersuchungen [Kinematoscopic investigations]. (1915). Zeitschrift fur Psycholigie 72, 194-296.

Kramer, P., \& Yantis, S. (1997). Perceptual grouping in space and time: evidence from the Ternus display. Perception \& Psychophysics, 59(1), 87-99.

Larsen A, Farrell JE, Bundesen C (1983) Short- and long-range processes in visual apparent movement

Ma-Wyatt, A., Clifford, C. W., \& Wenderoth, P. (2005). Contrast configuration influences grouping in apparent motion. Perception, 34(6), 669-685.

McBeath, M. K., Morikawa, K., \& Kaiser, M. K. (1992). Perceptual bias for forward-facing motion. Psychological Science, 3(6), 362-367.

Odic, D., Pratt, J. (2008). Solving the correspondence problem within the Ternus display: the differential-activation theory. Perception, 37(12), 1790-1804.

Pantle, A., \& Picciano, L. (1976). A multistable movement display: evidence for two separate motion systems in human vision. Science, 193(4252), 500-502.

Petersik, J. T., \& Pantle, A. (1979). Factors controlling the competing sensations produced by a bistable stroboscopic motion display. Vision Research, 19(2), 143-154.

Pikler, J. (1917). Sinnesphysiologische Untersuchungen. Leipzig: Barth. 
Proffit, D. R., Gilden, D. L., Kaiser, M. K., \& Whelan, S. M. (1988). The effect of configural orientation on perceived trajectory in apparent motion. Perception \& psychophysics, 43(5), 465-474.

Ramachandran, V.S., \& Anstis, S.M. (1986) The perception of apparent motion. Scientific American, 254, 102-109.

Shiffrar, M., \& Freyd, J.J. (1990). Apparenty Motion of the Human Body. Psychological Science, $1(4), 257-264$.

Ternus, J. (1926). Experimentelle untersuchungen uber phanomenale identitat (Experimental investigations of phenomenal identity). Psychologische Forschung, 7, 81-136.

Yu, K. (2000). Can semantic knowledge influence motion correspondence? Perception, 29, 693707. 